\title{
Rheumatic Immuno-related Adverse Events following Immunotherapy with Check- point Inhibitors: Adverse Drug Reaction, or Other?
}

\section{Ciro Manzo}

Azienda Sanitaria Locale Napoli 3 sud, Internal and Geriatric Medicine Department - Geronthorheumatological Outpatient Clinic, Poliambulatorio "Mariano Lauro", Sant'Agnello, Naples, Italy

\section{Dear Editors,}

I read with interest the case report entitled "Arthritis and myositis in a patient with programmed cell death-1 (PD-1) inhibitor pembrolizumab for lung cancer", recently published in the Mediterranean Journal of Rheumatology. ${ }^{1}$ Since 2011, when the Food and Drug Administration (FDA) approved the use of ipilimumab - a fully human monoclonal antibody against cytotoxic-T-lymphocyte antigen-4 (CTLA4) - for patients with metastatic melanoma, the immunotherapy with checkpoint inhibitors has been recommended for an increasing variety of cancers, both in metastatic and adjuvant settings.

It is a common knowledge that the same mechanism of action of these immune check-point inhibitors (ICls) can favor immune-related adverse events (IRAEs) which can affect multiple organ systems, and this risk is higher when two ICls are used in combination. Triggered by the growing use of ICls, an increasingly wide range of rheumatologic IRAEs have been described: arthritis, myositis, Sjogren's syndrome, polymyalgia rheumatica (PMR) or PMR-like syndromes, among these..$^{2-4}$

How were these rheumatologic IRAEs evaluated as an adverse drug reaction (ADR)? According to our recent systematic review regarding PMR and PMR-like syndromes, ${ }^{5}$ in most cases,

\section{Corresponding Author:}

Ciro Manzo

Poliambulatorio "Mariano Lauro"

Viale dei Pini 1

80065 Sant'Agnello, Naples, Italy

Tel.: +390815331465

Fax: +39 0815331449

E-mail: manzoreumatologo@libero.it only clinical judgment was used. Results were not very different when other different rheumatologic IRAEs were evaluated. ${ }^{6-8}$ This is a relevant discussion point, because within the field of pharmacovigilance, clinical judgment is often inferior to more structured methods of decision making that use simple algorithms. ${ }^{9} \mathrm{~A}$ number of algorithms have been proposed and some of these have been validated, but none of these have yet been accepted as gold standard. One commonly used algorithm is the ADR Probability Scale developed in 1981 by Naranjo and colleagues to standardize causality assessments. ${ }^{10}$ This scale classifies the probability that an adverse event is related to drug therapy. Naranjo's scale is based on a list of 10 weighted questions, which examine factors such as the temporal association of drug administration and event occurrence, alternative causes if any, drug levels, and previous patient experience with the same drug. The key advantages of this scale are its simplicity of use and clarity, and a significant increase in inter- and intra-rater agreement compared with standard clinical examination alone. The sum of the scores ranges from -4 to +13 , and is interpreted to reflect the strength of the probability that a drug has caused an ADR rather than the complication being a manifestation of the disease. A score $>9$ is empirically defined as "definitely" having caused the ADR; a score between 5 and 8 indicates that the drug "probably" caused the ADR; scores between 1 and 4 indicate that the ADR was "possibly" caused by the drug; and a score $<1$ indicate a "doubtful" association with the drug.

Recently, an EULAR task force confirmed that using this scale may help to assess the casual link between a specific rheumatic feature and ICls therapy. ${ }^{11}$ However, when, using reported data, we were able to apply Naranjo's scale to patients diagnosed with PMR following ICls therapy, their total scores were almost never $>4$. Moreover, a significant number of the patients improved without $\mathrm{ICl}$ discontinuation, and there was no relapse when $\mathrm{ICl}$ was re-introduced. ${ }^{5}$ 
In the initially mentioned case report, arthritis and myositis appeared 5 and half months after pembrolizumab initiation. ${ }^{1}$ In general, rheumatologic IRAEs can appear several months after immunotherapy initiation. This is due in part to ICls pharmacodynamic characteristics. Are rheumatic IRAEs following ICls therapy ADRs, or other?

It is common knowledge that arthritis, myositis, or other rheumatic inflammatory manifestations can be paraneoplastic findings. In cancer patients on $\mathrm{ICls}$, this possibility should carefully be assessed and excluded through a follow-up. A few researchers proposed that this follow-up should last no less than two years. ${ }^{12}$ Besides, some rheumatic manifestations could be a coincidence, and not induced by ICls therapy. For instance, an anti-citrullinated peptides antibodies (ACPA)-positive arthritis was reported in cancer patients following $\mathrm{ICls}$ therapy. ${ }^{13}$ Also, patients' response to therapy is unpredictable: in some cases, ICls discontinuation was necessary, but when ICls were reintroduced, rheumatologic IRAEs rarely reappeared. $^{14}$

In conclusion, is the methodological approach of deductive type we found in most published literature the appropriate one? Are we going in the right direction?

\section{CONFLICT OF INTEREST}

The author declares no conflict of interest.

\section{REFERENCES}

1. Pagkopoulou E, Simopoulou T, Maragkouli E, Perifanou-Sotiri S, Kotsakis A, Bogdanos DP. Arthritis and myositis in a patient treated with programmed cell death-1 (PD-1) inhibitor pembrolizumab for lung cancer. Mediterr J Rheumatol 2020;31:355-7.

2. Kostine M, Rouxel L, Barnetche T, Veillon R, Martin F, Dutriaux C, et al. Rheumatic disorders associated with immune checkpoint inhibitors in patients with cancer-clinical aspects and relationship with tumour response: a single-centre prospective cohort study. Ann Rheum Dis 2018;77:393-8.

3. Gediz F, Kobak S. Immune Checkpoint Inhibitors-related Rheumatic Diseases: What Rheumatologist Should Know? Curr Rheumatol Rev 2019;15:201-8.

4. Ramos-Casals M, Alexandre M, Suarez-Almazor ME, Lambotte O, Fisher BA, Hernandez-Molina G, et al. Sicca/Sjogren's syndrome triggered by PD-1/PD-L1 checkpoint inhibitors. Data from the International ImmunoCancer Registry (ICIR). Clin Exp Rheumatol 2019;37(Suppl 118):114-22.

5. Manzo C, Isetta M, Natale M, Castagna A. Identification and classification of polymyalgia rheumatica (PMR) and PMR-like syndromes following immune checkpoint inhibitors (ICls) therapy : discussion points and grey areas emerging from a systematic review of published literature. Medicines (Basel) 2020;7(11):68.

6. Richter MD, Crowson C, Kottschade LA, Finnes HD, Markovic SN, Thanarajasingam U. Rheumatic syndromes associated with immune checkpoint inhibitors: a single-center cohort of sixty-one patients. Arthritis Rheumatol 2019;71:468-75.

7. Kuswanto WF, MacFarlane LA, Gedmintas L, Mulloy A, Choueiri TK, Bermas BL. Rheumatologic symptoms in oncologic patients on PD-1 inhibitors. Semin Arthritis Rheum 2018;47:907-10.

8. Lidar M, Giat E, Garelick D, Horowitz Y, Amital H, Steinberg-Silman $Y$, et al. Rheumatic manifestations among cancer patients treated with immune checkpoint inhibitors. Autoimmun Rev 2018;17:2849.
9. Agbabiaka TB, Savovic J, Ernst E. Methods for causality assessment of adverse drug reactions. Drug Saf 2008;31:21-37.

10. Naranjo CA, Busto U, Sellers EM, Sandor P, Ruiz I, Robert EA, et al. A method for estimating the probability of adverse drug reaction. Clin Pharmacol Ther 1981;30:239-45.

11. Kostine M, Finckh A, Bingham 3rd CO, Visser K, Leipe J, Schulze-Koops $\mathrm{H}$, et al. EULAR points to consider for the diagnosis and management of rheumatic immune-related adverse events due to cancer immunotherapy with checkpoint inhibitors. Ann Rheum Dis 2020;0:1-13.

12. Azar L, Khasnis A. Paraneoplastic rheumatologic syndromes. Curr Opin Rheumatol 2013;25:44-9.

13. Leipe J, Christ LA, Arnoldi AP, Mille E, Berger F, Heppt M, et al. Characteristics and treatment of new-onset arthritis after checkpoint inhibitor therapy. RMD Open 2018;4:e000714.

14. Jamal S, Hudson M, Fifi-Mah A, Ye C. Immune-related adverse events associated with cancer immunotherapy: a review for the practicing rheumatologist. J Rheumatol 2020;47:166-75. 